\title{
Chaoization of Permanent Magnet Synchronous Motors Using Stator Flux Regulation
}

\author{
Zheng Wang, K. T. Chau, and Linni Jian \\ Department of Electrical and Electronic Engineering, The University of Hong Kong, Hong Kong, China
}

\begin{abstract}
In this paper, a new chaotic permanent magnet synchronous motor (PMSM) drive is proposed and implemented. The key is to apply the stator flux regulation (SFR) based direct torque control to intentionally chaoize the PMSM in such a way that chaotic motion can be resulted. A nonlinear speed controller, namely the sliding mode speed controller, is proposed to generate the torque reference for the torque controller. The proposed method not only offers the desired chaotic motion, but also provides the feature of controllable boundary. Both simulation and experimentation are given to confirm the validity.
\end{abstract}

Index Terms-Chaoization, direct torque control, flux regulation, permanent magnet motor.

\section{INTRODUCTION}

B ECAUSE of the random-like but bounded nature, chaos has been positively utilized in many industrial applications [1]. Rather than using mechanical means to generate chaotic motion for some niche applications such as mixing and compaction, electrical chaoization of motors takes the definite advantages of lightweight and compact size. A design-oriented approach was developed to chaoize a doubly salient permanent magnet (DSPM) motor in [2], and this chaotic DSPM motor was further applied for soil compaction in [3]. Although this design-oriented chaoizing approach can spontaneously produce chaotic motion once power on, it is inflexible since the motion boundary can not be tuned once the motor is designed. In [4], a time-delay feedback control approach was developed to chaoize a DC motor for liquid mixing. Although the motion boundary in [4] can be controlled by tuning the time-delay feedback control parameters, the corresponding torque response is sluggish. Also, the use of DC motors for industrial application is inefficient and needs regular maintenance.

The purpose of this paper is to propose and implement the stator flux regulation (SFR) based direct torque control approach for chaoization of the permanent magnet synchronous motor (PMSM). Thus, this control-oriented chaoizing approach will offer the definite advantages of fast torque response, controllable chaotic boundary, inherently high efficiency and maintenance-free operation.

\section{TORQUe CONTROLLER BASED ON SFR}

For a PMSM with no saliency, the electromagnetic torque $T$ can be expressed as $(3 / 2)\left(n_{p} / L_{s}\right)\left|\psi_{s} \| \psi_{f}\right| \sin \delta$ where $n_{p}$ is the number of pole pairs, $L_{s}$ is the stator inductance, $\psi_{s}$ is the stator flux, $\psi_{f}$ is the rotor flux, and $\delta$ is the angle between $\psi_{s}$ and $\psi_{f}$. Under the steady state, $\delta$ is a constant value, and the corresponding $\psi_{s}$ and $\psi_{f}$ rotate at synchronous speeds. Meanwhile, $\delta$ is a varying value under the transient state, and the corresponding $\psi_{s}$ and $\psi_{f}$ rotate at asynchronous speeds. Since the mechanical time constant of the rotor is much larger than the

Digital Object Identifier 10.1109/TMAG.2008.2002403

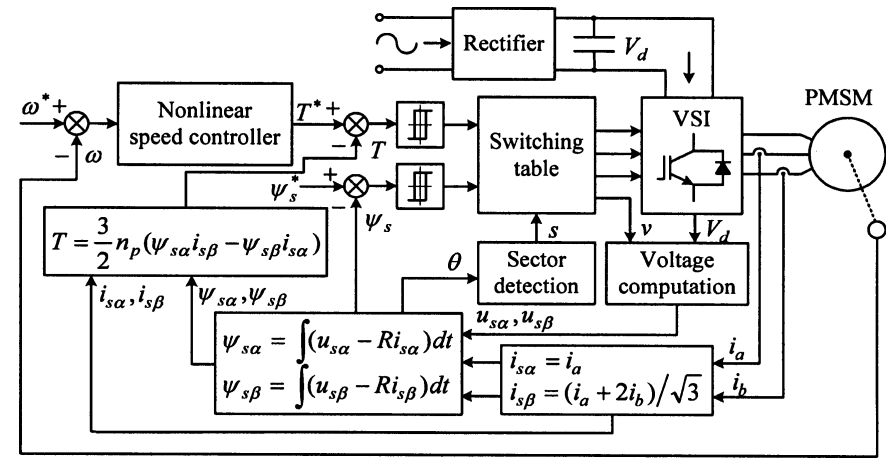

Fig. 1. Direct torque control based on stator flux regulation.

electrical time constant, $\psi_{f}$ can be considered to be stationary during stator flux regulation. Consequently, $T$ can be directly controlled by regulating the $\psi_{s}$ [5]. When $\left|\psi_{s}\right|$ is regulated to be constant during the operation, $T$ can be controlled by $\delta$ which will be realized by regulating the angle of $\psi_{s}$.

Fig. 1 shows the block diagram of direct torque control based on SFR. The measured voltages and currents are transformed into the stationary $\alpha$ and $\beta$ axes which are orthogonal to each other. The location of $\alpha$ axis aligns with that of the phase-A armature winding. Based on the PMSM model, $\psi_{s}$ can be estimated by using $u_{s \alpha}, u_{s \beta}, i_{s \alpha}$ and $i_{s \beta}$, while $T$ can be calculated by using $i_{s \alpha}, i_{s \beta}$ and the estimated $\psi_{s}$. Then, the regulation of $\psi_{s}$ is realized by selecting proper voltage space vectors. Fig. 2 shows the principle of selection of voltage space vectors for SFR under anti-clockwise motion. It is determined by the section where $\psi_{s}$ locates, and the outputs of two hysteresis loops for torque and flux. Since no current regulation and voltage modulation are needed, this direct torque controller based on SFR can provide fast torque response [6]. Thus, it is highly suitable to generate the desired fast-changing chaotic motion.

\section{NONLINEAR SPEED CONTROLlER}

In the proposed control scheme, the reference torque $T^{*}$ of the torque controller is generated by a nonlinear speed controller, namely a sliding mode speed controller. The sliding mode speed controller introduces a piecewise unit to directly act on the motor speed, which will facilitate generation of chaotic motion. This controller can offer some distinct features, such as fast dynamic response as well as robustness to load changes, 


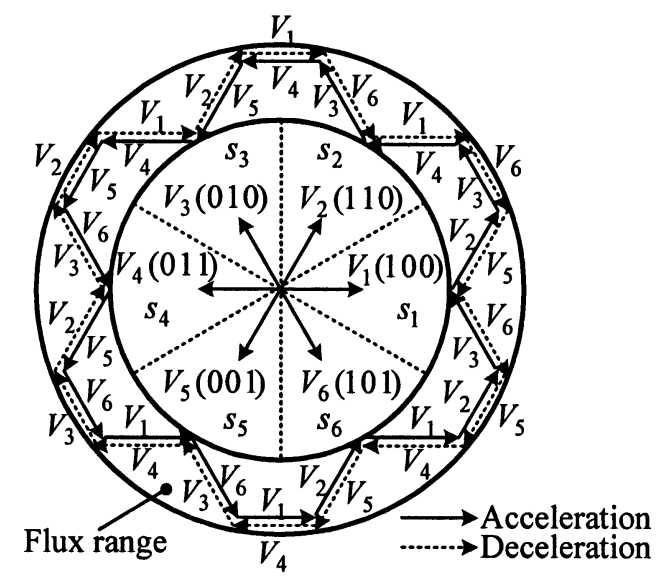

Fig. 2. Selection of switching status for stator flux regulation.

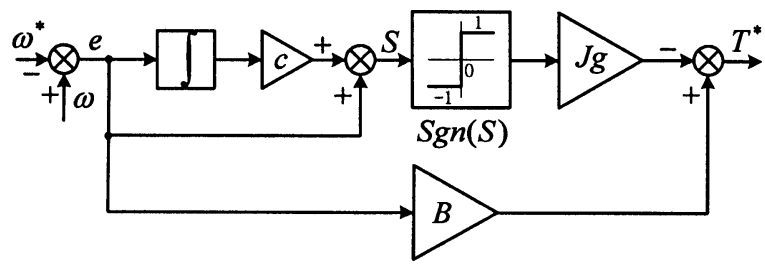

Fig. 3. Block diagram of sliding mode speed controller.

system disturbances and parameter variations. However, its control function is discontinuous on the sliding surface, which will cause chattering at certain control parameters and sampling rate $T_{s}$ [7]. Different from the sliding mode control in previous literature where the chattering is to be suppressed, this paper aims to positively utilize the nonlinear effect of hysteresis loop in the SFR based torque controller with sliding mode control. Hence, a large value of sampling rate $T_{s}$ is chosen so that it can intentionally generate a chattering $T^{*}$, and thus chaotic motion in the PMSM.

Fig. 3 shows the block diagram of the proposed sliding mode control. By defining the sliding surface $S$ as $\omega-\omega^{*}+c \int_{0}^{t}(\omega-$ $\left.\omega^{*}\right) d \tau$, the control law can be derived as $T^{*}=-J g \operatorname{sgn}(S)+$ $B \omega$ where $J$ is the rotor inertia, $B$ is the viscous damping, $c$ is a positive control parameter, and $g$ should be large enough to satisfy $g>\left|c\left(\omega-\omega^{*}\right)\right|$. This enables $S$ to converge to zero in the presence of rapid switching as given by the term $J g \operatorname{sgn}(S)$. Also, by properly choosing the control parameters for SFR such as the hysteretic flux boundary, the chaotic motion with controllable boundary can be realized.

\section{Simulation Results}

The performance of the whole system is simulated by MATLAB Simulink. A practical three-phase Sanyo Denki PMSM is used for exemplification. The key motor parameters for the proposed chaoization are listed in Table I. Firstly, the torque response of the SFR based torque controller is examined. In the torque controller, by choosing the stator flux reference $\psi_{s}^{*}=0.0344 \mathrm{~Wb}$, hysteretic torque boundary $\Delta T=0.005 \mathrm{Nm}$, hysteretic flux boundary $\Delta \psi=0 \mathrm{~Wb}$ and DC-link voltage $V_{d}=21 \mathrm{~V}$, the simulated waveforms of $T^{*}$ and $T$ are shown in Fig. 4(a) and (b), respectively. The
TABLE I

KEY MOTOR PARAMETERS

\begin{tabular}{|c|c|c|}
\hline Symbol & Quantity & Value \\
\hline$n_{p}$ & Number of pole pairs & 4 \\
\hline$\Psi_{f}$ & PM flux & $0.0344 \mathrm{~Wb}$ \\
\hline$R$ & Stator resistance & $4.3 \Omega$ \\
\hline$L_{s}$ & Stator inductance & $6.0 \mathrm{mH}$ \\
\hline$J$ & Rotor inertia & $7 \times 10^{-6} \mathrm{Nm} /\left(\mathrm{rad} / \mathrm{s}^{2}\right)$ \\
\hline$B$ & Viscous damping & $6.88 \times 10^{-6} \mathrm{Nm} /(\mathrm{rad} / \mathrm{s})$ \\
\hline
\end{tabular}

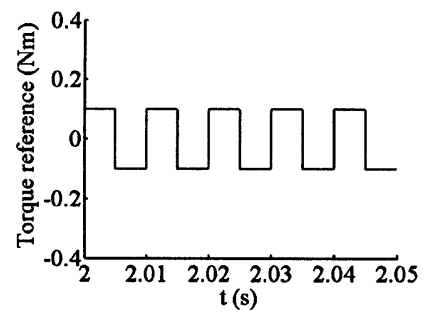

(a)

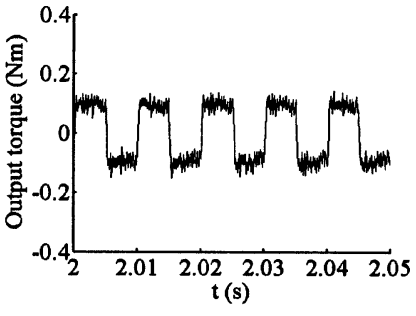

(b)
Fig. 4. Torque performance of torque controller: (a) torque reference; (b) output torque.

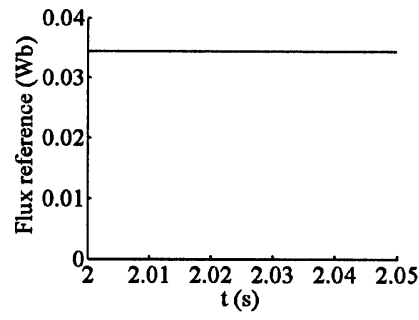

(a)

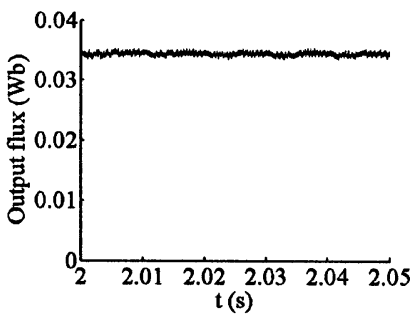

(b)
Fig. 5. Flux performance of torque controller: (a) stator flux reference; (b) output stator flux.

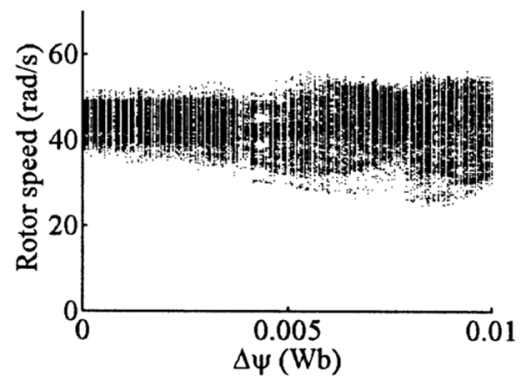

Fig. 6. Bifurcation diagram using sliding mode speed controller.

corresponding simulated waveforms of $\psi_{s}^{*}$ and $\psi_{s}$ are shown in Fig. 5(a) and (b), respectively. It can be seen that the output torque of the PMSM can track the rectangular torque reference quickly, while the stator flux can be regulated to follow a constant reference value. Hence, the torque controller based on SFR can provide fast torque response for electrical chaoization which is difficult to be achieved by the chaoizing approaches using current regulation and voltage modulation.

Secondly, the performance of chaoization using the sliding mode speed controller is examined by setting $g=1.43 \times 10^{7}$, $c=1$, and $T_{s}=100 \mu \mathrm{s}$. In order to evaluate the influence of $\Delta \psi$ on the chaotic motion boundary, the bifurcation diagram of the rotor speed of the PMSM against $\Delta \psi$ is plotted as shown in Fig. 6. The diagram is obtained by plotting the locally minimum values of rotor speed under different values of $\Delta \psi$. It can 


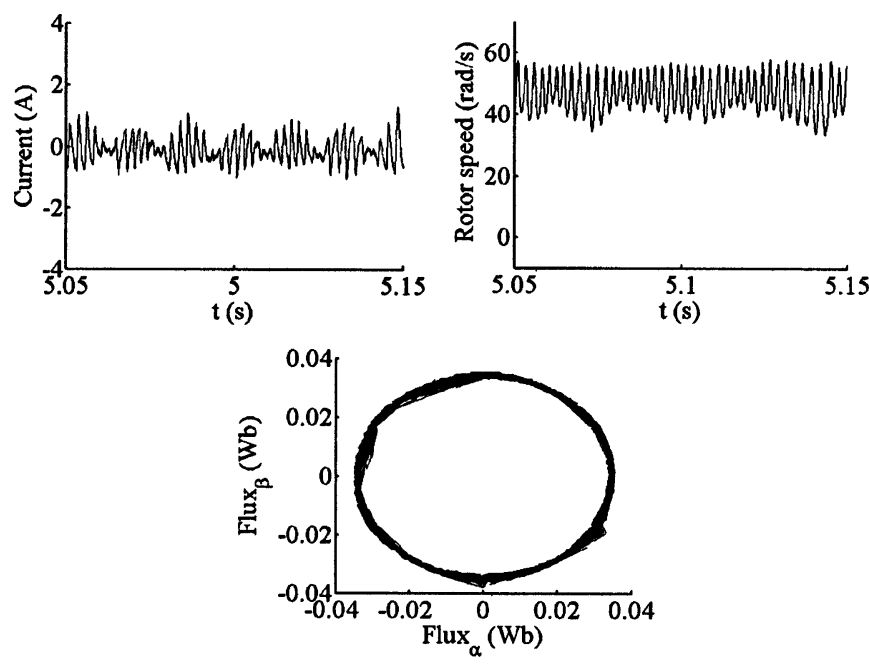

Fig. 7. Simulated results using sliding mode speed controller with $\Delta \psi=$ $0 \mathrm{~Wb}$.

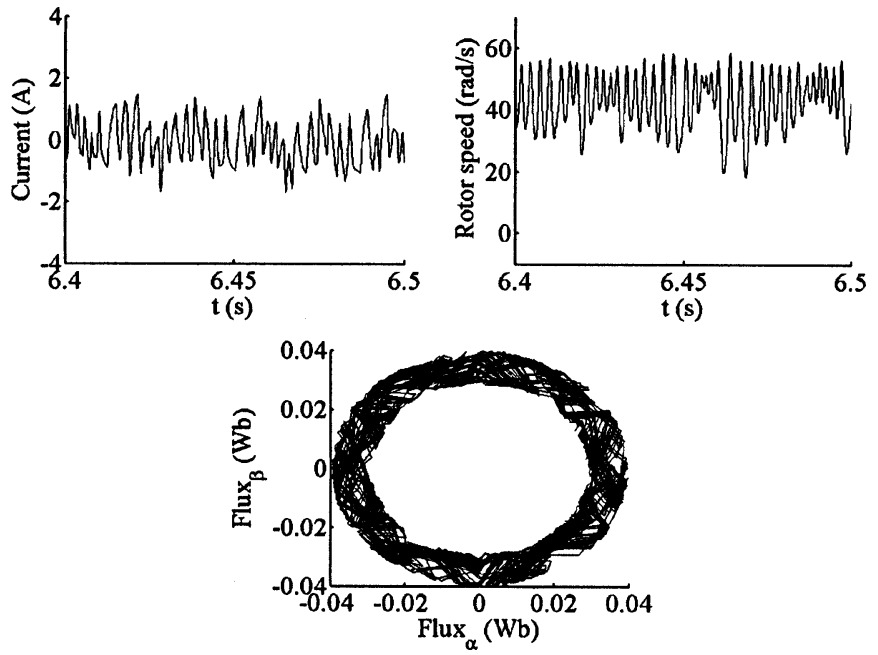

Fig. 8. Simulated results using sliding mode speed controller with $\Delta \psi=$ $0.01 \mathrm{~Wb}$.

be found that there are infinite values for the locally minimum rotor speed under each value of $\Delta \psi$. It illustrates that chaotic motion can be successfully generated, exhibiting random-like but bounded nature. Moreover, the chaotic boundary can be changed by tuning $\Delta \psi$ since different speed ranges under different values of $\Delta \psi$ can be observed in Fig. 6 . By setting $\Delta \psi$ as $0 \mathrm{~Wb}$, the waveforms of stator current and rotor speed as well as the trajectory of stator flux of the PMSM are simulated as shown in Fig. 7. Then, by changing $\Delta \psi$ to $0.01 \mathrm{~Wb}$, the corresponding simulated waveforms and trajectory are shown in Fig. 8. It confirms that the boundary of chaotic motion can be successfully controlled, which is an important feature that cannot be achieved by design-oriented chaoization.

\section{EXPERIMENTAL VERIFICATION}

In order to verify the proposed chaoization, the whole system is prototyped. A DSPACE DS1104 control board is used to implement the torque controller and speed controller digitally. The

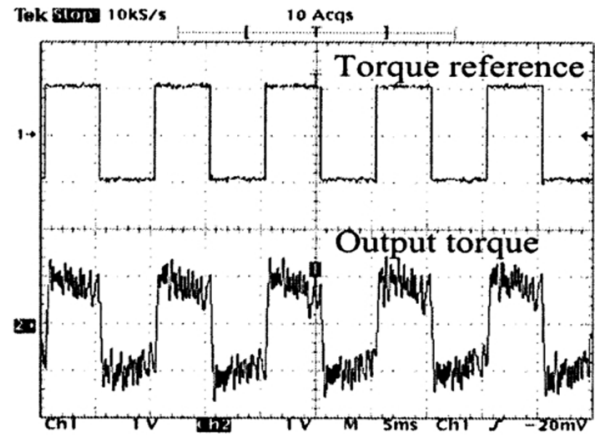

(a)

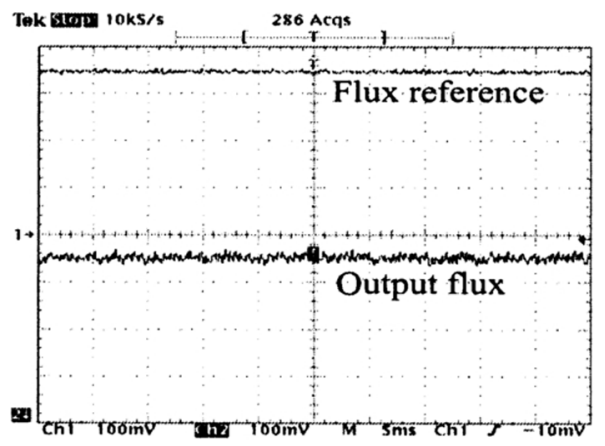

(b)

Fig. 9. Measured results of torque controller: (a) torque performance $(5 \mathrm{~ms} / \mathrm{div}$, $0.1 \mathrm{Nm} / \mathrm{div})$; (b) flux performance $(5 \mathrm{~ms} / \mathrm{div}, 0.01 \mathrm{~Wb} / \mathrm{div})$.

rotor speed is measured by the encoder on the motor and the current is measured by the sensor LEM LA25-NP, which are sampled by A/D on the control board at the rate of $100 \mu \mathrm{s}$. The control board supplies the switching signals for a three-phase IGBT-based voltage source inverter to drive the PMSM. Fig. 9 shows the measured torque and flux responses, which well agree with the simulated ones in Fig. 5, hence verifying the effectiveness of the SFR based torque controller experimentally. Figs. 10 and 11 show the measured waveforms and trajectories of the PMSM using the SFR based torque controller and the sliding mode speed controller when $\Delta \psi=0 \mathrm{~Wb}$ and $\Delta \psi=0.01 \mathrm{~Wb}$, respectively. They well agree with the simulated waveforms and trajectories. It should be noted that the corresponding chaotic patterns do not match in general, which is actually the randomlike nature of chaos. Nevertheless, the corresponding boundaries do closely match.

\section{CONCLUSION}

In this paper, the SFR-based direct torque control is used to provide fast torque response for chaoization of the PMSM in order to enhance some industrial applications such as compaction and mixing. A nonlinear speed controller, namely the sliding mode speed controller, is proposed to generate the desired chaotic motion. Computer simulations are carried out to illustrate that the proposed electrical chaoizing approach offers the definite advantages of fast torque response, controllable chaotic boundary, inherently high-efficiency and maintenance-free operation. In addition, experiments are given to verify the validity. 


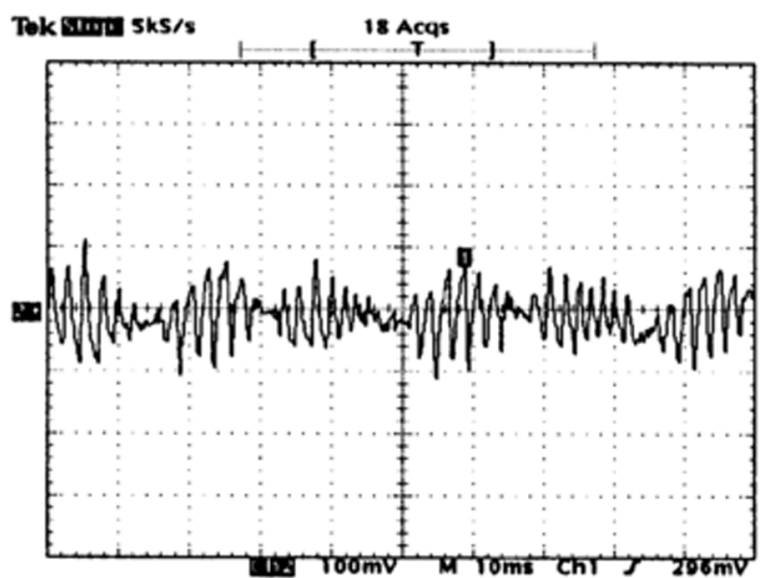

(a)

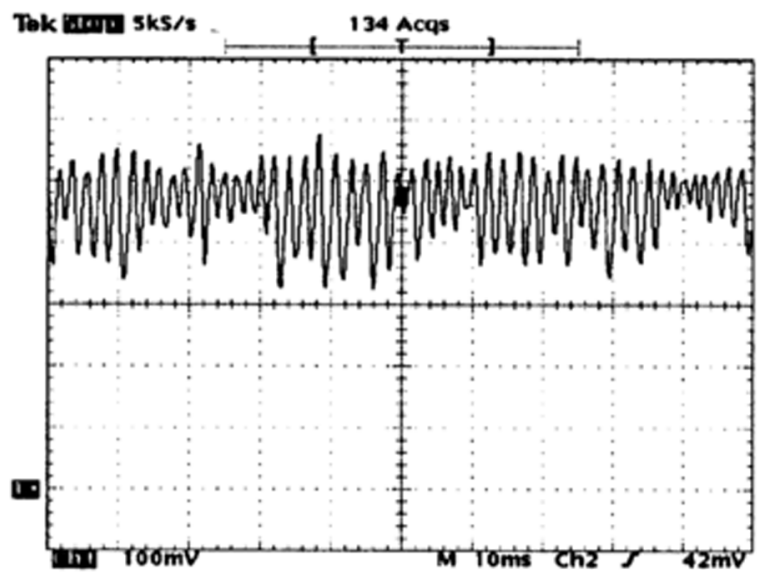

(b)

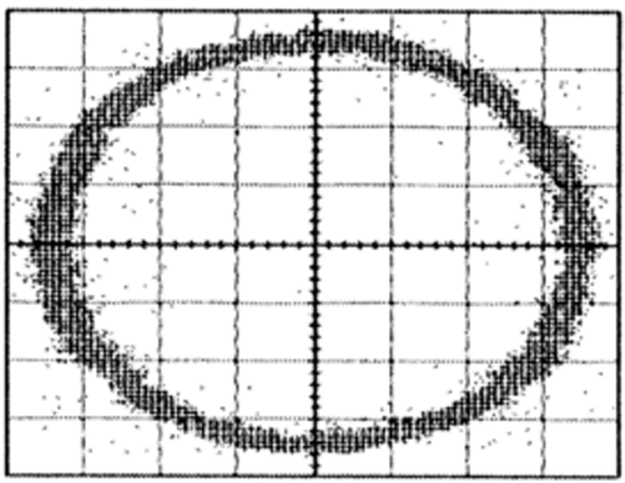

(c)

Fig. 10. Measured results using sliding mode speed controller with $\Delta \psi=0 \mathrm{~Wb}$ : (a) current waveform $(10 \mathrm{~ms} / \mathrm{div}, 1 \mathrm{~A} / \mathrm{div})$; (b) speed waveform $(10 \mathrm{~ms} / \mathrm{div}, 10 \mathrm{rad} / \mathrm{s} / \mathrm{div})$; (c) flux trajectory $(0.01 \mathrm{~Wb} / \mathrm{div} ; 0.01 \mathrm{~Wb} / \mathrm{div})$.

\section{ACKNOWLEDGMENT}

This work was supported by Project No. HKU7114/06E under the Research Grants Council, Hong Kong Special Administrative Region, China.

\section{REFERENCES}

[1] Z. Wang, K. T. Chau, and C. Liu, IEEE Trans. Magn., vol. 43, no. 6 , pp. 2612-2614, Jun. 2007.

[2] Y. Gao and K. T. Chau, IEEE Trans. Magn., vol. 40, no. 4, pp. 3038-3050, Jul. 2004.

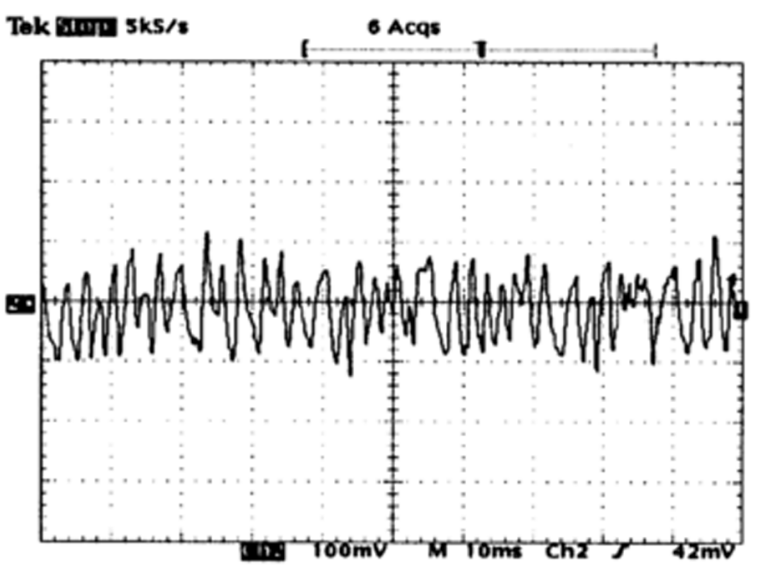

(a)

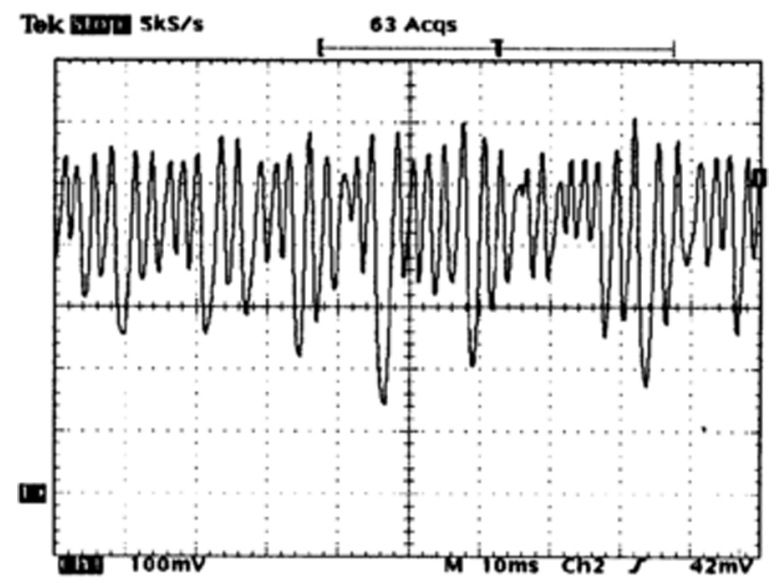

(b)

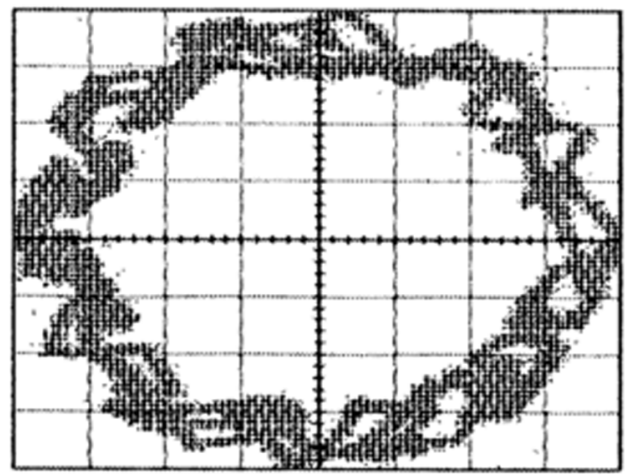

(c)

Fig. 11. Measured results using sliding mode speed controller with $\Delta \psi=0.01 \mathrm{~Wb}$ : (a) current waveform $(10 \mathrm{~ms} /$ div, $1 \mathrm{~A} / \mathrm{div})$; (b) speed waveform $(10 \mathrm{rad} / \mathrm{s} / \mathrm{div})$; (c) flux trajectory $(0.01 \mathrm{~Wb} / \mathrm{div} ; 0.01 \mathrm{~Wb} / \mathrm{div})$.

[3] K. T. Chau and Z. Wang, J. Appl. Phys., vol. 99, pp. 08R306:1-08R306:3, Apr. 2006.

[4] S. Ye and K. T. Chau, IEEE Trans. Ind. Electron., vol. 54, no. 4, pp. 2024-2032, Aug. 2007.

[5] M. F. Rahman, L. Zhong, M. E. Haque, and M. A. Rahman, IEEE Trans. Energy Convers., vol. 18, no. 1, pp. 17-22, Jan. 2003.

[6] P. Vas, Sensorless Vector and Direct Torque Control. Oxford, U.K.: Oxford Univ. Press, 1998.

[7] M. H. Park and K. S. Kim, IEEE Trans. Power Electron., vol. 6, no. 3, pp. 317-325, Jul. 1991.

Manuscript received March 01, 2008. Current version published December 17, 2008. Corresponding author: Z. Wang (e-mail: zwang@eee.hku.hk). 